\title{
Kinetic analysis of HER2-binding ABY-025 Affibody molecule using dynamic PET in patients with metastatic breast cancer
}

\author{
Ali Alhuseinalkhudhur ${ }^{1,2^{*}}$ (D, Mark Lubberink', Henrik Lindman², Vladimir Tolmachever, ${ }^{2,3}$ Fredrik Y. Frejd ${ }^{2,4}$, \\ Joachim Feldwisch ${ }^{2,4}$, Irina Velikyan ${ }^{1}$ and Jens Sörensen ${ }^{1}$
}

\begin{abstract}
Background: High expression of human epidermal growth factor receptor type 2 (HER2) represents an aggressive subtype of breast cancer. Anti-HER2 treatment requires a theragnostic approach wherein sufficiently high receptor expression in biopsy material is mandatory. Heterogeneity and discordance of HER2 expression between primary tumour and metastases, as well as within a lesion, present a complication for the treatment and require multiple biopsies. Molecular imaging using the HER2-targeting Affibody peptide ABY-025 radiolabelled with ${ }^{68} \mathrm{Ga}$-gallium for PET/CT is currently under investigation as a non-invasive tool for whole-body evaluation of metastatic HER2 expression. Initial studies demonstrated a high correlation between ${ }^{68} \mathrm{Ga}-\mathrm{ABY}-025$ standardized uptake values (SUVS) and histopathology. However, detecting small liver lesions might be compromised by high background uptake. This study aimed to explore the applicability of kinetic modelling and parametric image analysis for absolute quantification of ${ }^{68} \mathrm{Ga}-\mathrm{ABY}-025$ uptake and HER2-receptor expression and how that relates to static SUVs.

Methods: Dynamic ${ }^{68} \mathrm{Ga}-\mathrm{ABY}-025$ PET of the upper abdomen was performed 0-45 min post-injection in 16 patients with metastatic breast cancer. Five patients underwent two examinations to test reproducibility. Parametric images of tracer delivery $\left(K_{1}\right)$ and irreversible binding $\left(K_{i}\right)$ were created with an irreversible two-tissue compartment model and Patlak graphical analysis using an image-derived input function from the descending aorta. A volume of interest (VOI)-based analysis was performed to validate parametric images. SUVs were calculated from $2 \mathrm{~h}$ and $4 \mathrm{~h}$ post-injection static whole-body images and compared to $K_{i}$.
\end{abstract}

Results: Characterization of HER2 expression in smaller liver metastases was improved using parametric images. $K_{i}$ values from parametric images agreed very well with VOl-based gold standard $\left(R^{2}>0.99, p<0.001\right)$. SUVs of metastases at $2 \mathrm{~h}$ and $4 \mathrm{~h}$ post-injection were highly correlated with $K_{i}$ values from both the two-tissue compartment model and Patlak method $\left(R^{2}=0.87\right.$ and 0.95 , both $\left.p<0.001\right) .{ }^{68} \mathrm{Ga}-\mathrm{ABY}-025$ PET yielded high testretest reliability (relative repeatability coefficient for Patlak 30\% and for the two-tissue compartment model 47\%).

Conclusion: ${ }^{68} \mathrm{Ga}$-ABY-025 binding in HER2-positive metastases was well characterized by irreversible two-tissue compartment model wherein $K_{i}$ highly correlated with SUVs at 2 and $4 \mathrm{~h}$. Dynamic scanning with parametric image formation can be used to evaluate metastatic HER2 expression accurately.

Keywords: HER2 receptor, Metastatic breast cancer, Affibody, Dynamic PET, Kinetic modelling

\footnotetext{
* Correspondence: ali.alkhudhur@igp.uu.se

${ }^{1}$ Nuclear Medicine and PET, Department of Surgical Sciences, Uppsala

University, Uppsala, Sweden

Department of Immunology, Genetics and Pathology, Uppsala University,

Uppsala, Sweden

Full list of author information is available at the end of the article
} 


\section{Introduction}

The introduction of trastuzumab, a HER2-targeting monoclonal antibody, improved the outcome of HER2enriched breast cancer treatment dramatically $[1,2]$. Still, breast cancer is a heterogeneous disease and HER2 expression may vary considerably not only within a given lesion but also between the primary tumour and metastases [3-5]. Obtaining multiple biopsies in a patient is not always possible, and the need has emerged for a robust imaging technique to estimate HER2 status in cancer patients that is non-invasive, quantitative, and cost-effective and allows for repeated examinations.

One of the most successful classes investigated as HER2imaging agents is Affibody molecules [6]. The secondgeneration anti-HER2 Affibody molecules (code-named ABY-025) have high stability, diminished interaction with immunoglobulins, and picomolar affinity for HER2 as well as rapid pharmacokinetics with fast blood clearance, resulting in high tumour-to-background ratios shortly after injection [7-10]. In a pilot study, ${ }^{68}$ Ga-ABY-025 PET uptake quantification suggested a preliminary SUV threshold of 6 for the discrimination of HER2-positive from HER2negative lesions $[11,12]$. SUV is a highly repeatable measurement; however, it relies heavily on the acquisition and reconstruction protocols used [13].

SUV correlated strongly with IHC scores performed on biopsies. However, the liver had the second highest SUV values among normal tissue after the kidneys at 60 240 min post-injection with about $9 \%$ of the injected activity retained by the liver $[12,14]$. The relatively high liver background uptake might mask the signal from smaller liver metastases, especially those with low to moderate HER2-receptor expression, and that might compromise the accuracy of HER2 scoring by PET. The solution for the improved image analysis in the liver is crucial given that HER2-positive breast cancer subtypes have a higher probability of liver metastasis which, in these subtypes, is the second most common metastatic site, compared to HER2-negative subtypes [15].

SUV does not distinguish between specific and nonspecific uptake in receptor-binding radiotracers such as, in this case, ${ }^{68} \mathrm{Ga}$-ABY-025 $[16,17]$. A more accurate investigation of the underlying uptake properties requires kinetic modelling. The rate constants obtained from fitting the time-activity curves (TACs) using kinetic modelling can potentially be used to increase contrast by eliminating non-specific background activity found in surrounding tissue. This, in principle, could enhance tumour visualization in these organs $[18,19]$. Individual rate constants, or micro-parameters, describing the rate of transport of the tracer between plasma and tissue and between different states (e.g. free and internalized) in tissue can be estimated using compartment models. The gold standard for tracer kinetic analysis is the estimation of these rate constants by non-linear regression of the operational equations describing these compartment models. In case of a tracer with irreversible kinetics, these micro-parameters can then be used to estimate the net influx rate of the tracer, which, in the present case, is assumed to be related to the HER2. Compartment models can be simplified by linearization using basis functions, which allows for a fast calculation of rate constants for each voxel, resulting in parametric images showing the parameter of interest at the voxel level. A simplified linearized method is the Patlak method, which is a graphical analysis technique that allows for the estimation of the net influx rate without giving information on the individual rate constants.

The present study aimed to (1) explore the applicability of kinetic modelling and (2) parametric image analysis for absolute quantification of ${ }^{68} \mathrm{Ga}-\mathrm{ABY}-025$ uptake and HER2-receptor expression and (3) how that relates to static SUVs.

\section{Materials and methods \\ Patients}

This was a reanalysis of 16 patients with metastatic breast cancer, described in detail elsewhere [12]. Twelve had HER2-positive tumours and 4 had HER2-negative tumours, according to IHC and FISH. Nine patients of the HER2-positive group and all HER2-negative patients had liver metastases. Patient characteristics are presented in Table 1.

\section{Radiochemistry}

The GMP compliant manual production of low and high peptide dose ${ }^{68} \mathrm{Ga}-\mathrm{ABY}-025$ was published earlier [20].

Table 1 Patient characteristics

\begin{tabular}{lll}
\hline & $\begin{array}{l}\text { HER2-positive }(n=12), \\
\text { number of patients (\%) }\end{array}$ & $\begin{array}{l}\text { HER2-negative }(n=4), \\
\text { number of patients (\%) }\end{array}$ \\
\hline Median age & 61 & 66 \\
$\begin{array}{l}\text { ER-positive } \\
\text { Site of disease }\end{array}$ & $9(75 \%)$ & $3(75 \%)$ \\
Locoregional & $4(33 \%)$ & $2(50 \%)$ \\
Liver & $9(75 \%)$ & $4(100 \%)$ \\
Bone & $8(67 \%)$ & $3(75 \%)$ \\
Lung & $2(17 \%)$ & $1(25 \%)$ \\
Lymph nodes & $3(25 \%)$ & $1(25 \%)$ \\
CNS & $3(25 \%)$ & $2(50 \%)$ \\
Contralateral breast & 0 & $1(25 \%)$ \\
Other & $4(33 \%)$ & $1(25 \%)$ \\
Ongoing treatment & $11(92 \%)$ & 0 \\
with trastuzumab & & $3(75 \%)$ \\
Biopsy post-PET & $9(75 \%)$ &
\end{tabular}

Reprinted from [12] 
Briefly, five vials each containing $100 \mu$ g of GMP quality ABY-025 (Affibody AB) were used for the labelling synthesis that resulted in the high peptide dose (427 \pm $19 \mu \mathrm{g})$ radiopharmaceutical. The ${ }^{68} \mathrm{Ge} /{ }^{68} \mathrm{Ga}$ generator (1850 MBq IGG100, Eckert \& Ziegler) was thoroughly validated and qualified. Production and quality control were accomplished within $1 \mathrm{~h}$ with a high success rate $(100 \%)$ and radiochemical purity $(99.1 \pm 0.4 \%)$.

\section{PET protocol}

All 16 patients underwent 1 fluorodeoxyglucose ${ }^{18} \mathrm{~F}$ FDG PET (GE Discovery ST, GE Healthcare) examination within 14 days prior to the ${ }^{68} \mathrm{Ga}-\mathrm{ABY}-025$ PET. Patients were divided into 2 groups. The first group included 10 patients who underwent $1{ }^{68} \mathrm{Ga}$-ABY-025 PET examination, while the second group included 6 patients who underwent 2 examinations 1 week apart for a testretest study, and 1 patient had no detectable lesions; thus, no retest study was performed [12].

Each PET study for patients in both groups began with a 45-min dynamic scan $(6 \times 10,3 \times 20,3 \times 60,5 \times 180$, $5 \times 300$ s) starting simultaneously with the ${ }^{68} \mathrm{Ga}-\mathrm{ABY}-$ 025 intravenous injection $(241 \pm 49 \mathrm{MBq})$. Dynamic scans for all patients were performed over the abdomen, except for 2 patients who were scanned over the chest area considering that they had metastases within that area. Static whole-body scans from the skull to midthigh were performed at $2 \mathrm{~h}$ and $4 \mathrm{~h}$ post-injection for the first group ( 4 and $5 \mathrm{~min}$ per bed position, respectively), while the second group underwent only 1 static scan at $2 \mathrm{~h}$ post-injection. Images were reconstructed using settings supplied by the vendor (OSEM with 2 iterations and 21 subsets; corrections for dead time, decay, and attenuation) into a $128 \times 128$ matrix with voxel size $3.9 \times 3.9 \times 3.27 \mathrm{~mm}$. A post-processing Gaussian filter of $6 \mathrm{~mm}$ was applied, resulting in an image resolution of 7-8 $\mathrm{mm}$.

\section{Volumes of interest}

Volumes of interest (VOIs) were defined manually on the dynamic PET images using the Carimas 2.9 software (Turku PET Centre, Turku, FI). Concurrent CT and
FDG PET/CT scans were used to guide VOI definition. The defined VOIs included metastatic lesions in the liver and bone, lung, and lymph nodes, where available. VOIs were also defined over representative parts of healthy organs including the liver, bone marrow, spleen, kidney cortex, gastric ventricle, small intestines, myocardium, and lungs. The aorta was segmented in the frame where the first pass of the radioactivity peaked. The same VOIs were defined manually on the second dynamic PET for the second group of patients and on the $2 \mathrm{~h}$ and $4 \mathrm{~h}$ post-injection static images. Lesion margins were avoided where possible while defining VOIs to minimize both spill-in and spill-out partial volume effects. One patient was excluded from further analysis due to severe motion artefacts.

The total number of lesions defined in the first group of patients was 24, of which 12 were over $1 \mathrm{~cm}^{3}$ and 5 over $2 \mathrm{~cm}^{3}$. The total number of lesions defined for the second group was 16 , of which 8 were over $1 \mathrm{~cm}^{3}$ and 5 over $2 \mathrm{~cm}^{3}$.

\section{SUV}

SUVs from decay-corrected static PET study activity concentrations at $2 \mathrm{~h}$ and $4 \mathrm{~h}$ post-injection in all patients were calculated using Eq. (1):

$$
\mathrm{SUV}=\frac{\text { Activity conc. }\left(\frac{\mathrm{Bq}}{\mathrm{ml}}\right) \times \text { bodyweight }(\mathrm{g})}{\text { Injected activity }(\mathrm{Bq})}
$$

SUV mean values were used for comparison with parametric images values.

\section{Kinetic analysis}

A VOI-based analysis was performed using a MATLAB program written in-house. Data were analysed using non-linear regression of the operational equations of the single-tissue compartment model (1TC), the irreversible two-tissue compartment model (2TC-3k) (Fig. 1), and the reversible two-tissue compartment model (2TC-4k), all including blood volume as a fitted parameter, as well as the Patlak graphical analysis. The preferred compartment

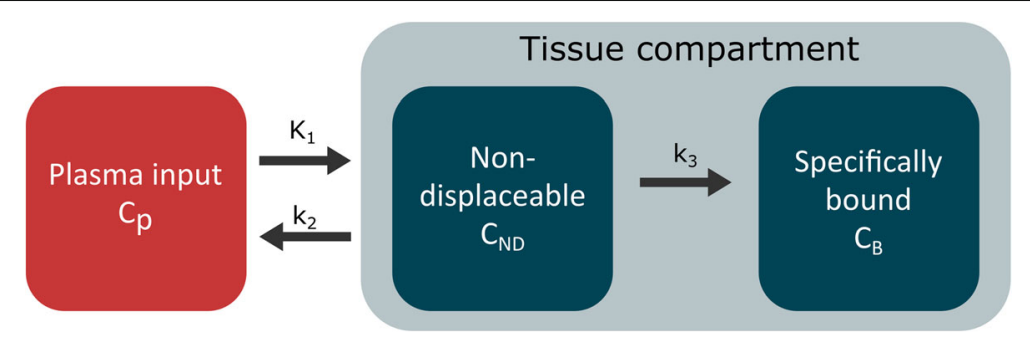

Fig. 1 Two-tissue compartment kinetic model used in the analysis of dynamic PET studies with ${ }^{68} \mathrm{Ga}-\mathrm{ABY}-025$ in patients with metastatic breast cancer. $K_{1}, k_{2}$, and $k_{3}$ represent the transfer rate constants 
model was established using the Akaike information criterion [21]. These models were used to calculate the rate constants including $K_{1}$, which is the delivery constant, and, for the 2TC models, $K_{i}$, which represent the uptake or influx rate constant. Aorta TACs were used as an input function (Fig. 2). The net influx rate constant was calculated with Eq. (2):

$$
K_{i}=\frac{K_{1} \times k_{3}}{k_{2}+k_{3}}
$$

The $K_{i}$ cut-off value for HER2 positivity corresponding to the previously defined cut-off SUV value of 6 [12] was determined, along with its confidence interval.

\section{Parametric images}

Parametric images were created using programs written in-house, with aortic TACs as input function. Parametric images of $K_{1}, K_{i}, V_{b}$, and $V_{\mathrm{ND}}=K_{1} / k_{2}$ (delivery, net influx rate, partial blood volume, and non-displaceable volume of distribution) were computed using a basis function implementation of the irreversible 2TC model [19]. In addition, $K_{i}$ and $V_{e}$ (distribution volume) images were computed using the Patlak method. The VOIs previously defined on the dynamic images were transferred to the parametric images to obtain the mean values of the kinetic parameters with each VOI.

\section{Image contrast}

For both SUV and parametric $K_{i}$ images, image contrast was evaluated as the ratio between values in the liver metastases and healthy liver tissue ( $\mathrm{T} / \mathrm{N}$ ratios).

\section{Statistical analysis}

The Mann-Whitney test was used to compare metastatic lesions with normal tissue unless otherwise specified. Wilcoxon's signed-rank $t$ test was used to compare the same parameters between the test and retest studies in the second group of patients. The Bland-Altman plot was created, and intraclass relative repeatability coefficient was calculated for the test-retest group. A $p$ value of less than 0.05 was considered statistically significant. Cut-off values were calculated using the best-fit linear regression equation between parametric image values and SUVs, and 95\% confidence intervals were calculated using the standard error values of the slope and intercept. All statistical analyses were performed using Prism 7 (GraphPad Software, Inc).

\section{Results}

The kinetics of ${ }^{68} \mathrm{Ga}-\mathrm{ABY}-025$ were best described by the irreversible two-tissue compartment model (2TC-3k model), giving the lowest Akaike information criterion in 22 out of 40 TACs (55\%). Patlak $K_{i}$ values showed an excellent agreement with 2TC $K_{i}$ values both for VOIbased analysis and in parametric images (Fig. 3a, b). Both Patlak and 2TC $K_{i}$ values derived from parametric images agreed very well to their counterparts obtained from the VOI-based analysis $\left(n=24, R^{2}>0.99\right.$, and $p<$ 0.001 for both correlations; Fig. 3c, d).

$K_{i}$ presented good contrast and low background uptake in the normal liver (Fig. 4a). $K_{i}$ values were 3.7 - and 7.1-fold higher in the metastatic lesions compared to the normal liver (Tmax/Nmean ratio 2TC, $3.7 \pm 2.8$; Patlak, $7.1 \pm 7.8)$. The Patlak Tmax/Nmean ratio was significantly higher $(p<0.05)$ than the corresponding SUV-based ratio $(4.2 \pm 3.4$ at $2 \mathrm{~h}$ post-injection). All metastases invariably had lower tracer delivery rates than normal liver and were

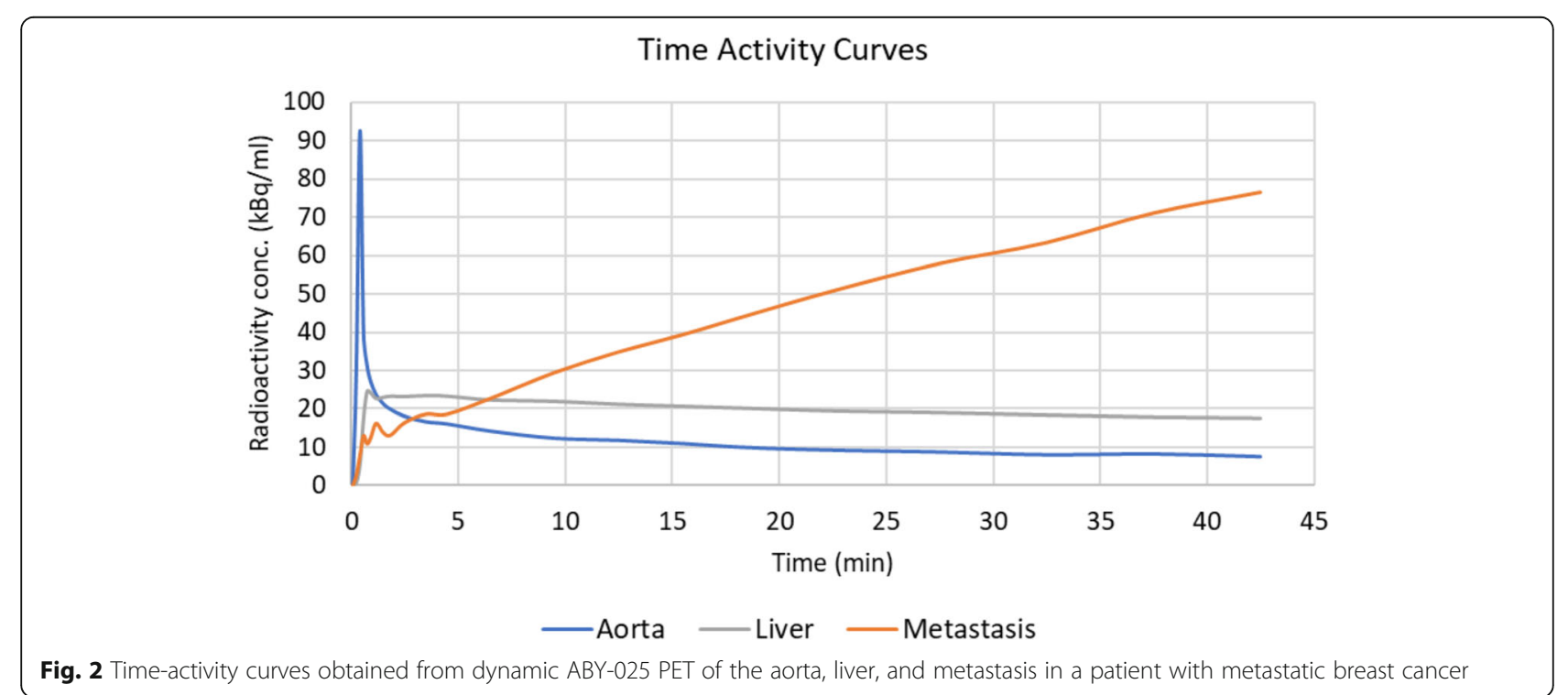



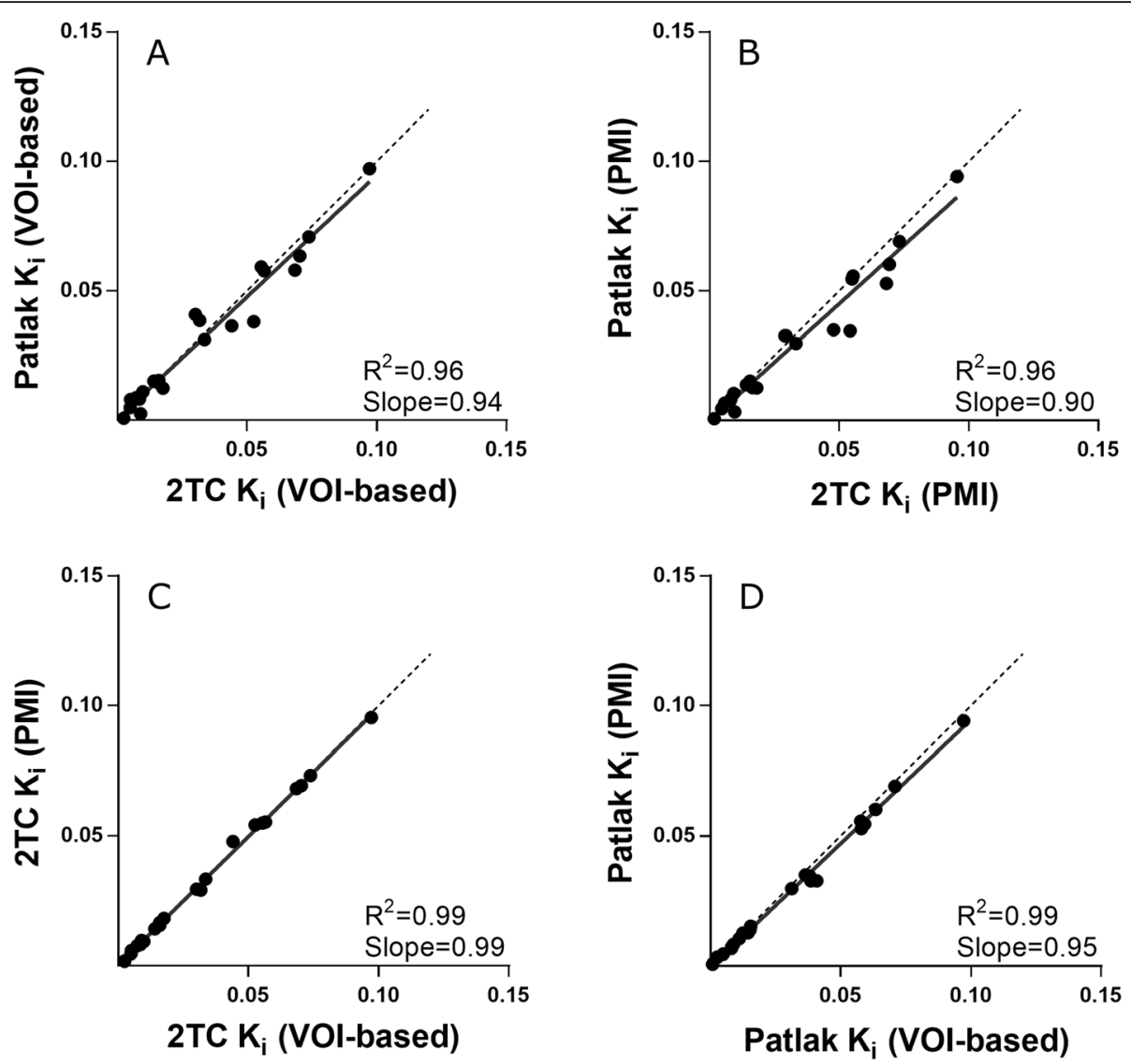

Fig. 3 Correlations between a VOI-based 2TC $K_{i}$ and Patlak $K_{i j}$ b parametric 2TC $K_{i}$ and Patlak $K_{i}$ c VOI-based and parametric 2TC $K_{i}$ values, and $\mathbf{d}$ VOI-based and parametric Patlak $K_{i}$ values (PMI, parametric images)

visualized as cold spots in $K_{1}$ images (Fig. 4a, b). Parametric image-based rate constant values and SUV for both liver metastases and normal liver are presented in Table 2.

${ }^{68} \mathrm{Ga}-\mathrm{ABY}-025$ binding in HER2-negative lesions was equal to or lower than the uptake in the normal liver in both $K_{i}$ and static SUV images (Fig. 4b). $K_{i}$ values of $0.015 \mathrm{~mL} / \mathrm{cm}^{3} / \mathrm{min}$ for $2 \mathrm{TC}-3 \mathrm{k}$ model and $0.013 \mathrm{~mL} /$ $\mathrm{cm}^{3} / \mathrm{min}$ for the Patlak model corresponded to the proposed SUV cut-off of 6.0 at $2 \mathrm{~h}$ post-injection [12], yielding 95\% confidence intervals of 4.4-7.6 (2TC) and 4.8-7.2 (Patlak).

Static SUVs obtained at $2 \mathrm{~h}$ and $4 \mathrm{~h}$ post-injection had a good correlation with parametric image-based $K_{i}$ (Patlak $R^{2}=0.95 ; 2 \mathrm{TC} R^{2}=0.87, n=9$, Fig. 5). T/N ratios were $4.2 \pm 3.4$ and $4.7 \pm 4.6$ for $2 \mathrm{~h}$ and $4 \mathrm{~h}$ post-injection, respectively $(n=10) .{ }^{68} \mathrm{Ga}$-ABY-025 PET test-retest reliability was high as shown by the parametric image-based $K_{i}$ values in the test-retest group of patients (Pearson $r \geq$ $0.92, n=5$ ) with a relative repeatability coefficient of $30 \%$ for Patlak $K_{i}$ and $47 \%$ for 2 TC $K_{i}$ compared to $32 \%$ for SUV at $2 \mathrm{~h}$ (Fig. 6). Neither systematic nor proportional bias was observed in the test-retest group (Fig. 7).

\section{Discussion}

In this study, we assessed the kinetics of ${ }^{68} \mathrm{Ga}-\mathrm{ABY}-025$ with the hypotheses that visualization and determination of HER2 status in liver metastases could be facilitated by eliminating background uptake through creating parametric images of the tracer uptake kinetics, and that simple static SUVs in tumours are acceptable surrogates for the true binding kinetics of the tracer.

The 2TC-3k irreversible model best fitted the data according to the Akaike information criterion. This coincided with the preclinical findings showing almost irreversible kinetic properties of Affibody molecules in HER2-expressing tumours [22]. Since the majority of the measurements in patients were taken from liver lesions, the heterogeneity induced by spill-in from gradually lower uptake in normal liver probably explains why there was a small reversible component in a subset of the measurements.

Both VOI-based kinetic modelling and parametric image analysis returned virtually identical $K_{i}$ values $\left(R^{2}=0.99, n=\right.$ $24)$, indicating that VOI-based kinetics are reproducible on the voxel level. The basis function implementation of the 

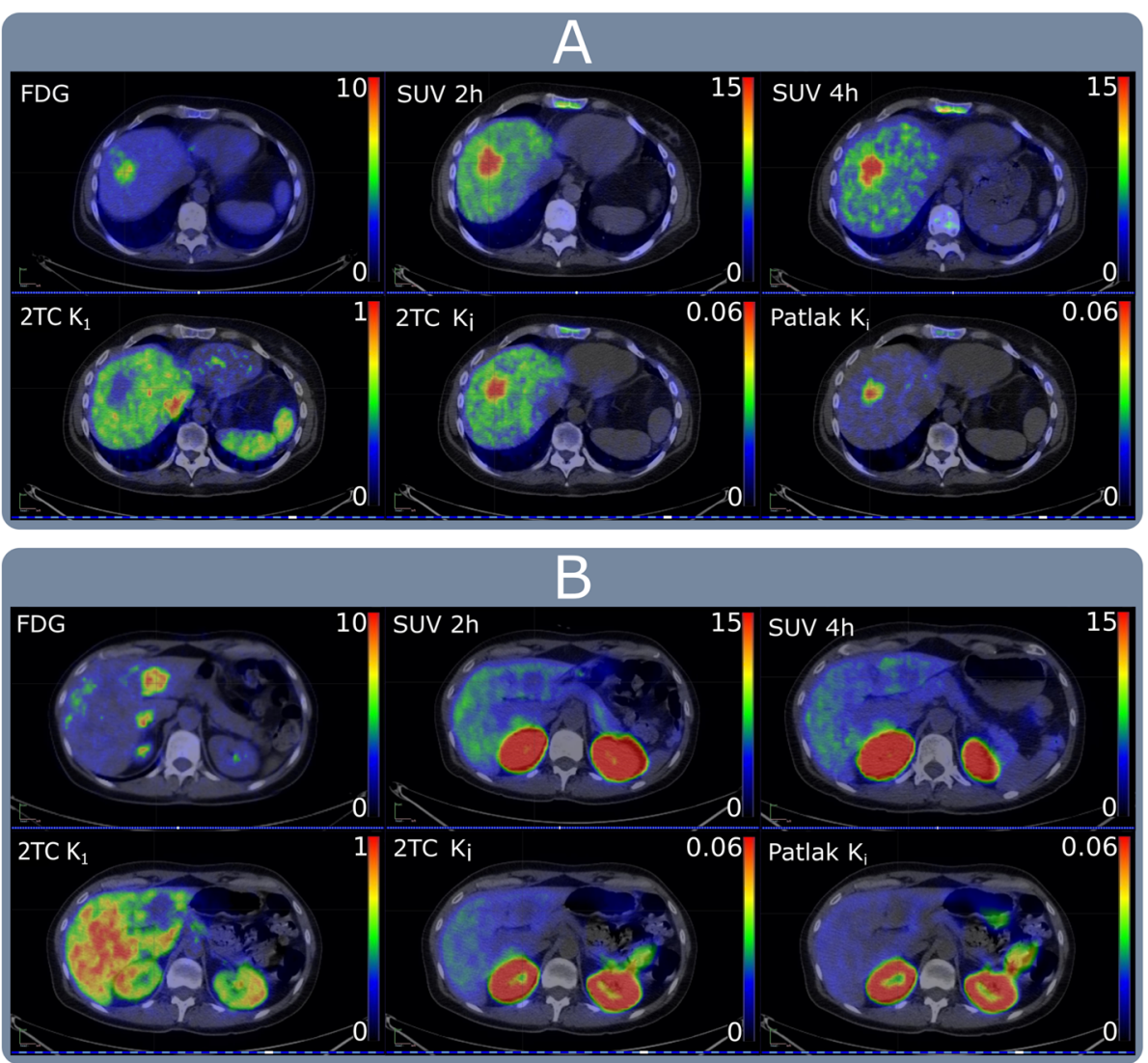

Fig. 4 Positron emission tomography (PET) images. a In a patient with HER2-positive expression. b In a patient with HER2-negative expression. ${ }^{18} \mathrm{~F}-\mathrm{FDG}$ PET images to the left followed by SUV static image taken at $2 \mathrm{~h}$ time point after ${ }^{68} \mathrm{Ga}-\mathrm{ABY}-025$ injection. $2 \mathrm{TC} K_{i}$, Patlak $K_{i,}$ and $(2 \mathrm{TC}) K_{1}$ are parametric images created from the ${ }^{68} \mathrm{Ga}-\mathrm{ABY}-025$ dynamic PET study $\left(K_{1}\right.$, transfer rate constant from plasma to the tissue compartment; $K_{i r}$ influx rate of the tracer into irreversible compartment)

2TC-3k model also provided $K_{1}$ images that reflected the delivery rate of the tracer to various tissues, including tumours. This is important when estimating the fraction of the tracer available to cancer lesions for the subsequent computation of the tracer net influx rate $K_{i}$.

There was a strong correlation between tumour static SUV values and $K_{i}$ values (Patlak $R^{2}=0.95 ; 2$ TC $R^{2}=$ $0.87, n=9$, Fig. 5), indicating that the static SUV images provided a close representation to HER2-specific binding since parametric $K_{i}$ images reflect the specific uptake solely and eliminate any non-specific binding that increases background uptake. The relatively narrow 95\% confidence intervals, especially with Patlak $K_{i}$, around the proposed SUV cut-off of 6 uphold its use in the upcoming clinical trials and provide a reference to the degree of uncertainty paired with it. Moreover, there was a very good correlation in the test-retest group (Fig. 6), with a relative repeatability coefficient of $30 \%$ for Patlak model, indicating high reproducibility of kinetic analysis and similar to the results with static SUV values demonstrated previously [12]. These findings further support the claim that SUV values can be used for the quantification of HER2 expression, as concluded in our previous paper [12]. This will be used in a large multicentric phase II/III clinical trial for non-invasive quantification of HER2 expression in advanced breast cancer using ${ }^{68} \mathrm{Ga}-\mathrm{ABY}-025$ PET (NCT03655353) that is currently ongoing.

Table $2{ }^{68} \mathrm{Ga}-\mathrm{ABY}-025$ SUVs and parametric image values in normal liver and liver metastasis, median (range)

\begin{tabular}{llllll}
\hline & SUV $2 \mathrm{~h}$ & SUV $4 \mathrm{~h}$ & $2 \mathrm{TC}-3 \mathrm{k} K_{1}$ & $2 \mathrm{TC}-3 \mathrm{k} K_{i}$ & Patlak $K_{i}$ \\
\hline Normal liver & $4.9(3-6.7)$ & $4.8(3.8-6.1)$ & $0.37(0.20-0.56)$ & $0.015(0.006-0.03)$ & $0.008(0.004-0.016)$ \\
All liver met & $10(3.1-32.4)$ & $10(3.6-39)$ & $0.20(0.02-0.38)$ & $0.025(0.009-0.095)$ & $0.025(0.003-0.094)$ \\
$p$ value* $^{*}$ & $<0.0001$ & 0.008 & 0.0001 & 0.019 & 0.0002 \\
\hline
\end{tabular}

SUV standardized uptake value, $2 T C$ - $3 k$ irreversible two-tissue compartment model, $K_{i}$ transfer rate constant

*Mann-Whitney-Wilcoxon test 

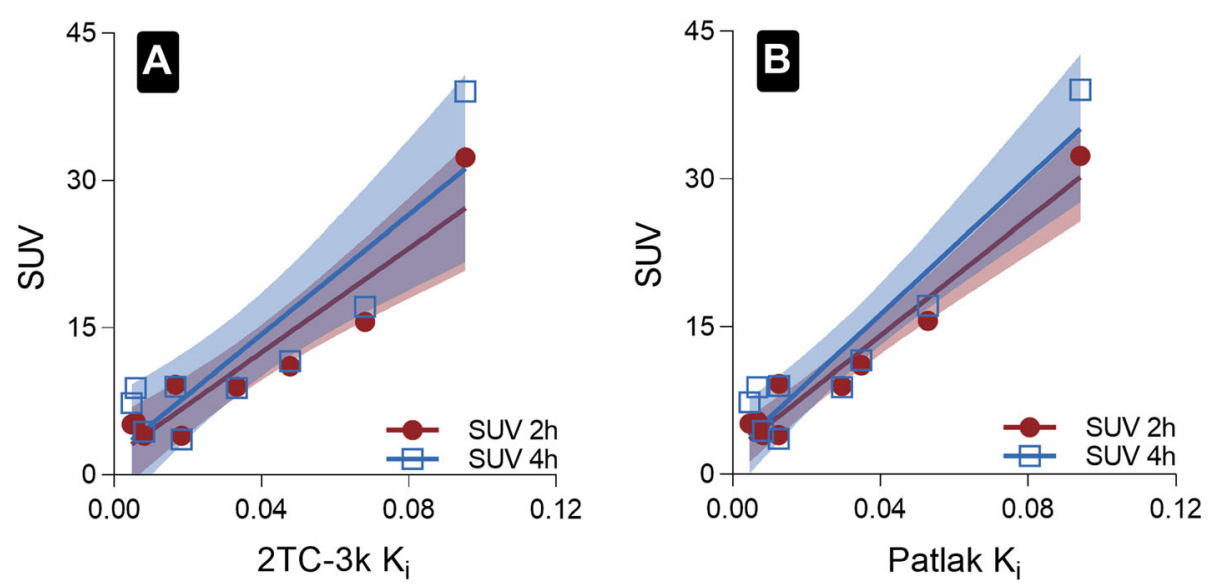

Fig. 5 a 2 TC-3K $K_{i}$ correlation with SUV $2 \mathrm{~h}$ and SUV $4 \mathrm{~h}\left(R^{2}=0.87,0.80\right.$ respectively, both $\left.p<0.0001\right)$. b Patlak $K_{i}$ correlation with SUV $2 \mathrm{~h}$ and SUV $4 \mathrm{~h}\left(R^{2}=0.95,0.90\right.$ respectively, both $\left.p<0.0001\right)$. Shaded areas represent $95 \%$ confidence band

A noticeable degree of specific uptake was recognized in normal liver, further supporting the claim that HER2 receptors are expressed in normal liver tissue [23], although at levels significantly lower than in HER2positive tumours. The Human Protein Atlas describes liver HER2 expression as moderate, compared to other normal tissues [24]. Liver retention in static PET images is much higher than what can be accounted for by specific binding. This was explained by the very high first-pass extraction with $K_{1}$ values significantly higher than for all tumours, probably related to the specialized non-continuous endothelium of liver capillaries. While visualization of the HER2-expressing lesions was possible in both parametric $K_{i}$ and static SUV images (Fig. 4a), with the feasibility to localize small liver metastases, tumour-to-liver ratios were significantly higher in parametric Patlak $K_{i}$ images. Thus, parametric imaging might become beneficial in a few clinical cases where it might be difficult to grade the HER2 expression in small metastases due to the high liver background uptake. Because dynamic imaging introduces an additional 45 -min scan, the presence of small suspicious liver lesions $(<1 \mathrm{~cm})$ should be known from other imaging modalities beforehand and the resulting HER2 score should be of direct relevance for therapeutic decisions. In terms of contrast, the Patlak method performed better than the 2TC-3k method, mainly because $K_{i}$ values in the normal liver were 30\% lower with the Patlak method than with the 2TC-3k model, while the Patlak distribution volume $\left(V_{e}\right)$ values were $30 \%$ higher than those based on the $2 \mathrm{TC}-3 \mathrm{k}$ model $\left(V_{\mathrm{ND}}\right)$. The kinetics of ${ }^{68} \mathrm{Ga}-\mathrm{ABY}-025$ in the liver are not irreversible, which leads to an underestimation of $K_{i}$ and an overestimation of $V_{e}$ when using the Patlak method, and an additional reason of the higher Patlak distribution volume is that it includes blood volume, whereas $V_{\mathrm{ND}}$ does not. Hence, the improved contrast using Patlak $K_{i}$ is
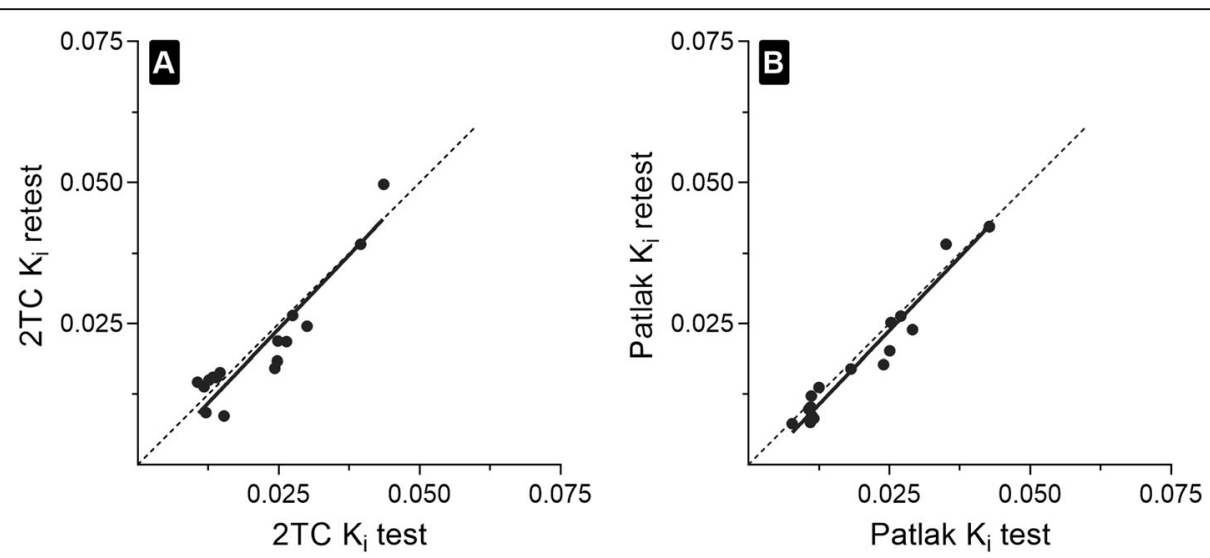

Fig. 6 Reproducibility of parametric images shown as a correlation of a test-retest 2 TC $K_{i}$ values $\left(R^{2}=0.85, p<0.0001\right)$ and $\mathbf{b}$ test-retest Patlak $K_{i}$ values $\left(R^{2}=0.94, p<0.0001\right)$ for the metastatic lesions in a group of patients rescanned after 1 week $(n=5)$. The solid line is the Deming line, and the dotted line is the identity line 


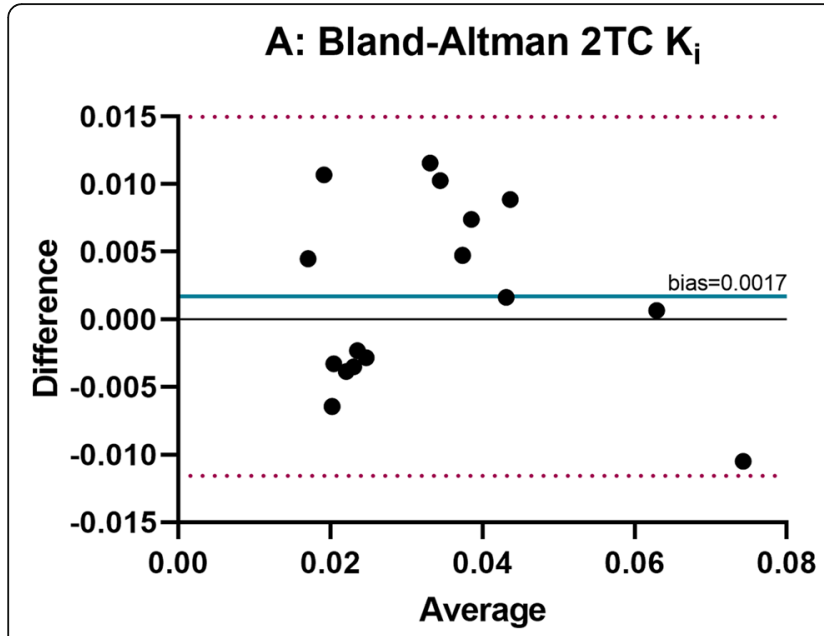

B: Bland-Altman Patlak $\mathbf{K}_{\mathbf{i}}$

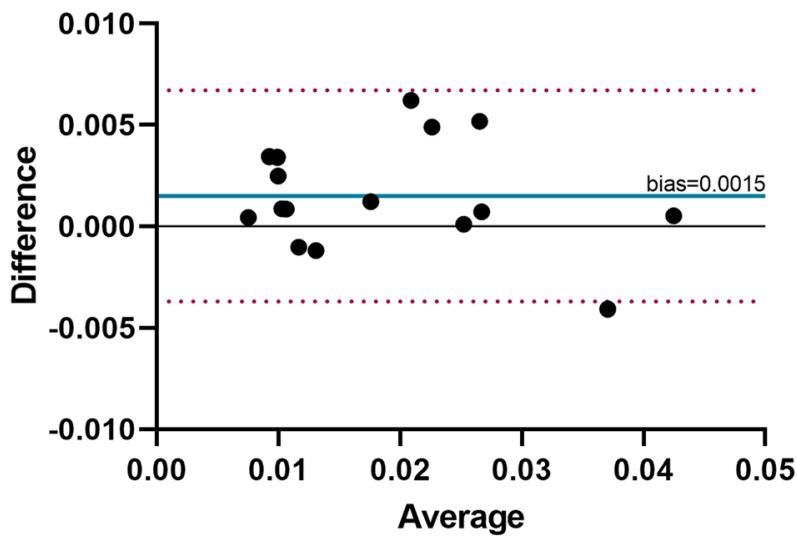

Fig. 7 The Bland-Altman plot a of two-tissue compartment influx rate (2TC-3K $\left.K_{i}\right)$ and $\mathbf{b}$ Patlak $K_{i}$

essentially a fortunate benefit of the erroneous assumption of irreversible kinetics in healthy liver tissue, likely resulting in better lesion detection in Patlak $K_{i}$ images than in SUV images, as clearly shown in Fig. 8 . Most peptidebased PET tracers are retained and metabolized in the liver, and as a result, non-specific liver uptake is a common problem in PET imaging. This phenomenon is also observed in recent biodistribution studies [25]. Similarly, preclinical studies investigating the use of other Affibody molecules targeting HER3 receptors also observed non-specific uptake of the given tracer in the liver [26]. Parametric image formation with the enhanced tumour-to-liver ratio is a favourable workaround to this issue not limited to ABY-025 PET but could potentially establish a framework for future studies with similar tracers.
HER2 imaging provides an extended scale to the degree of expression contrary to traditional IHC that incorporates the entire range of positive lesions into the ordinal HER2 $3+$, as shown in the previous studies [11, 12, 27].

The main limitation of the study is the small number of subjects. Data was used to explore multiple aims, which increases the risk of type 1 errors. There were few lesions with a definite diagnosis from biopsies, and only few patients had HER2-negative disease status. A substantial fraction of lesions were small, which introduces measurement errors. Dynamic studies in the present work were limited to a single bed position, allowing for only $15 \mathrm{~cm}$ axial field of view. Multi-bed dynamic PET in current clinical scanners or research total-body PET scanners with automated definition of the input function

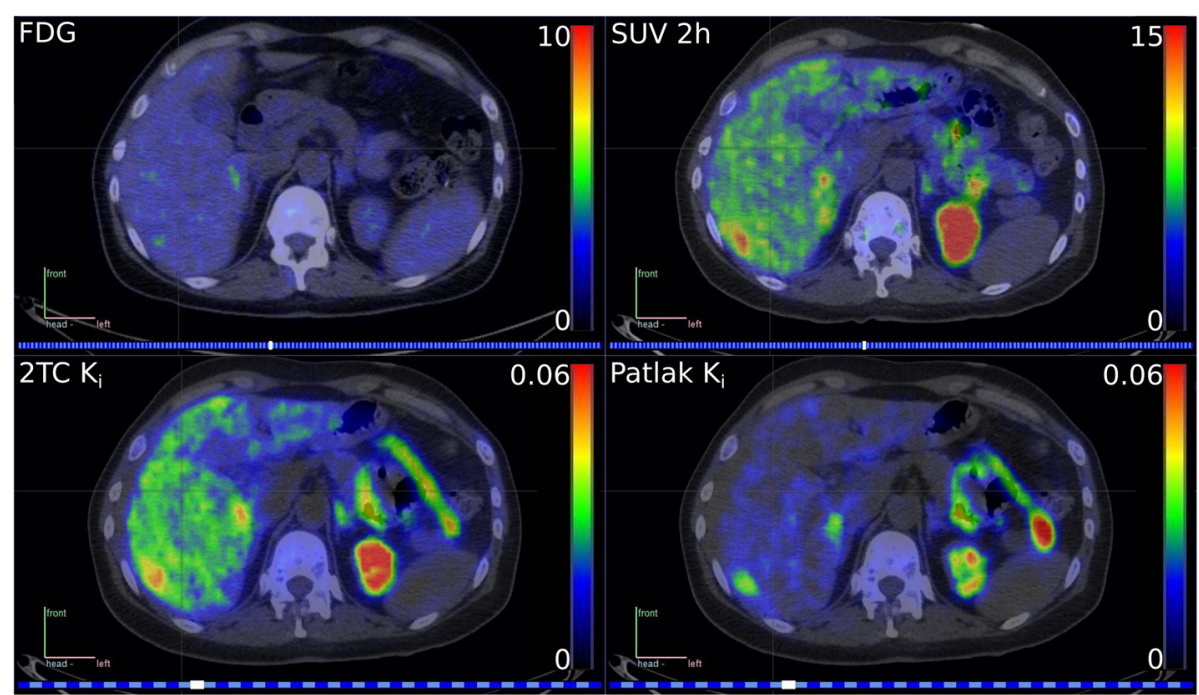

Fig. 8 FDG PET and ABY-025 PET SUV $2 \mathrm{~h}$ and parametric images of both Patlak $K_{j}$ and 2 TC-3k $K_{j}$ in a breast cancer patient with multiple small liver metastases 
could allow for performing whole-body parametric imaging and correlative analysis of SUV and T/B ratios towards parametric imaging of the entire body [28, 29]. Future studies should also investigate if the dynamic protocol could be shortened.

\section{Conclusion}

Parametric imaging provided good visualization and eliminated non-specific background uptake in the liver. There was a good correlation of absolute quantification presented by kinetic parameters and the simple SUV from static $2 \mathrm{~h}$ and $4 \mathrm{~h}$ time point images, further supporting the use of SUV for clinical routine in whole-body imaging and HER2expression quantification. Besides, $K_{1}$ and $K_{i}$ images provided insight on the pharmacokinetics of ${ }^{68} \mathrm{Ga}-\mathrm{ABY}-025 . K_{i}$ values might be useful for the quantification of HER2 expression in breast cancer patients under certain conditions, such as anti-HER2 drug development studies and in some clinical cases with known small tumours in the liver. Further studies with a larger patient cohort and more biopsies are warranted.

\section{Abbreviations}

1TC: Single-tissue compartment; 2TC-3k: Irreversible two-tissue compartment; 2TC-4k: Reversible two-tissue compartment; FISH: Fluorescence in situ hybridization; GMP: Good manufacturing practice;

IHC: Immunohistochemistry; $K_{1}$ : Delivery rate; $K_{i}$ : Net influx rate; OSEM: Ordered Subsets Expectation-Maximization; PET/CT: Positron emission tomography/computed tomography; PMI: Parametric images; SPECT: Singlephoton emission computed tomography; SUV: Standardized uptake value; T/ N: Tumour-to-normal tissue; TAC: Time-activity curve; $V_{b}$ : Partial blood volume; $V_{e}$ : Distribution volume; $V_{\mathrm{ND}}$ : Non-displaceable volume of distribution; VOI: Volume of interest

\section{Acknowledgements}

The authors wish to thank the staff at Uppsala PET centre and Department of Nuclear medicine for their great help in this project.

\section{Authors' contributions}

AA performed the image analysis and statistical analysis and contributed largely to the writing of the manuscript and preparation of the figures. ML designed and wrote the MATLAB scripts used in VOl-based analysis and in creating parametric images, assisted in the image and statistical analysis, and reviewed the manuscript. HL attributed to the conception of the study plan, supervised the data collection and image analysis, and reviewed the manuscript. VT attributed to the conception of the study plan and reviewed the manuscript. FYF and JF assisted in the data collection, contributed to the manufacturing of the Affibody molecule, and reviewed the manuscript. IV supervised and assisted in the radiolabelling of the Affibody, contributed to the statistical analysis, and reviewed the manuscript. JS designed the study, supervised the image analysis, and contributed to the writing and finalization of the manuscript and figures. All authors read and approved the final manuscript.

\section{Funding}

This work was sponsored by The Swedish Cancer Society (Cancerfonden) and The Swedish Breast Cancer Foundation (Bröstcancerfonden). ABY-025 was provided by Affibody AB. FF and JF are employees of Affibody AB. No other potential conflicts of interest relevant to this article exist. Open access funding provided by Uppsala University.

\section{Availability of data and materials}

The datasets used and/or analysed during the current study are available from the corresponding author on reasonable request.

\section{Ethics approval and consent to participate}

The clinical trial (EudraCT 2012-005228-14 and NCT01858116) was approved by the Regional Ethics Review Board in Uppsala (Dnr 2012/554), Radiation Protection Ethics Committee (Dnr D13/01), and Swedish Medical Products Agency (Dnr 151:2012/121491) [11]. All patients signed informed consent before inclusion in the study.

\section{Consent for publication}

All patients signed informed consent for publication before inclusion in the study.

\section{Competing interests}

The authors declare that they have no competing interests.

\section{Author details}

${ }^{1}$ Nuclear Medicine and PET, Department of Surgical Sciences, Uppsala University, Uppsala, Sweden. ${ }^{2}$ Department of Immunology, Genetics and Pathology, Uppsala University, Uppsala, Sweden. ${ }^{3}$ Research Centrum for Oncotheranostics, Research School of Chemistry and Applied Biomedical Sciences, Research Tomsk Polytechnic University, Tomsk, Russia. ${ }^{4}$ Affibody AB, Solna, Sweden.

\section{Received: 8 November 2019 Accepted: 31 January 2020}

\section{0}

\section{References}

1. Baselga J, Arteaga CL. Critical update and emerging trends in epidermal growth factor receptor targeting in cancer. J Clin Oncol. 2005;23:2445-59.

2. Yarden Y, Sliwkowski MX. Untangling the ErbB signalling network. Nat Rev Mol Cell Biol. 2001;2:127-37.

3. Wolff AC, Hammond MEH, Schwartz JN, Hagerty KL, Allred DC, Cote RJ, et al. American Society of Clinical Oncology/College of American Pathologists guideline recommendations for human epidermal growth factor receptor 2 testing in breast cancer. J Clin Oncol. 2006;25:118-45.

4. Houssami N, Macaskill P, Balleine RL, Bilous M, Pegram MD. HER2 discordance between primary breast cancer and its paired metastasis: tumor biology or test artefact? Insights through meta-analysis. Breast Cancer Res Treat. 2011;129:659-74

5. Cottu PH, Asselah J, Lae M, Pierga J-Y, Diéras V, Mignot L, et al. Intratumoral heterogeneity of HER2/neu expression and its consequences for the management of advanced breast cancer. Ann Oncol. 2008;19:595-7.

6. Frejd FY, Kim K-T. Affibody molecules as engineered protein drugs. Exp Mol Med. 2017;49:e306

7. Orlova A, Wållberg H, Stone-Elander S, Tolmachev V. On the selection of a tracer for PET imaging of HER2-expressing tumors: direct comparison of a 124l-labeled Affibody molecule and trastuzumab in a murine xenograft model. J Nucl Med. 2009:50:417-25.

8. Orlova A, Tolmachev V, Pehrson R, Lindborg M, Tran T, Sandström M, et al. Synthetic Affibody molecules: a novel class of affinity ligands for molecular imaging of HER2-expressing malignant tumors. Cancer Res. 2007:67:2178-86.

9. Löfblom J, Feldwisch J, Tolmachev V, Carlsson J, Ståhl S, Frejd FY. Affibody molecules: engineered proteins for therapeutic, diagnostic and biotechnological applications. FEBS Lett. 2010;584:2670-80.

10. Feldwisch J, Tolmachev V, Lendel C, Herne N, Sjöberg A, Larsson B, et al. Design of an optimized scaffold for Affibody molecules. J Mol Biol. 2010;398: 232-47

11. Sörensen J, Sandberg D, Sandström M, Wennborg A, Feldwisch J, Tolmachev $V$, et al. First-in-human molecular imaging of HER2 expression in breast cancer metastases using the $111 \mathrm{ln}$-ABY-025 Affibody molecule. J Nucl Med. 2014;55:730-5.

12. Sörensen J, Velikyan I, Sandberg D, Wennborg A, Feldwisch J, Tolmachev V, et al. Measuring HER2-receptor expression in metastatic breast cancer using [68Ga]ABY-025 Affibody PET/CT. Theranostics. 2016:6:262-71.

13. Lodge MA. Repeatability of SUV in oncologic 18F-FDG PET. J Nucl Med. 2017;58:523-32.

14. Sandström M, Lindskog K, Velikyan I, Wennborg A, Feldwisch J, Sandberg D, et al. Biodistribution and radiation dosimetry of the anti-HER2 Affibody molecule Ga-68-ABY-025 in breast cancer patients. J Nucl Med. 2016;57:867-71. 
15. Wu Q, Li J, Zhu S, Wu J, Chen C, Liu Q, et al. Breast cancer subtypes predict the preferential site of distant metastases: a SEER based study. Oncotarget. 2017:8:27990-6.

16. Kinahan PE, Fletcher JW. PET/CT standardized uptake values (SUVs) in clinical practice and assessing response to therapy. Semin Ultrasound CT MR. 2010;31:496-505.

17. Yoder KK. Basic PET data analysis techniques. In: Misciagna S, editor. Positron Emiss Tomogr - Recent Dev Instrum Res Clin Oncol Pract [Internet]. Rijeka: InTech; 2013 [cited 2017 Feb 9]. p. 63-80. Available from: http:// www.intechopen.com/books/positron-emission-tomography-recentdevelopments-in-instrumentation-research-and-clinical-oncological-practice/ basic-pet-data-analysis-techniques.

18. Cherry SR, Sorenson JA, Phelps ME. Tracer kinetic modeling. Phys Nucl Med [Internet]. 4th ed. Philadelphia: W.B. Saunders; 2012 [cited 2016 May 23]. p. 379-405. Available from: http://www.sciencedirect.com/science/article/pii/ B9781416051985000216.

19. Karakatsanis NA, Lodge MA, Tahari AK, Zhou Y, Wahl RL, Rahmim A. Dynamic whole body PET parametric imaging: I. Concept, acquisition protocol optimization and clinical application. Phys Med Biol. 2013;58: $7391-418$

20. Velikyan I, Wennborg A, Feldwisch J, Lindman H, Carlsson J, Sörensen J. Good manufacturing practice production of [68Ga]Ga-ABY-025 for HER2 specific breast cancer imaging. Am J Nucl Med Mol Imaging. 2016;6:135-53.

21. Akaike H. A new look at the statistical model identification. IEEE Trans Autom Control. 1974;19:716-23.

22. Trousil S, Hoppmann S, Nguyen Q-D, Kaliszczak M, Tomasi G, Iveson P, et al. Positron emission tomography imaging with 18F-labeled ZHER2:2891 Affibody for detection of HER2 expression and pharmacodynamic response to HER2-modulating therapies. Clin Cancer Res. 2014;20:1632-43.

23. Pai-Scherf LH, Villa J, Pearson D, Watson T, Liu E, Willingham MC, et al. Hepatotoxicity in cancer patients receiving erb-38, a recombinant immunotoxin that targets the erbB2 receptor. Clin Cancer Res. 1999;5: 2311-5.

24. Uhlén M, Fagerberg L, Hallström BM, Lindskog C, Oksvold P, Mardinoglu A, et al. Tissue-based map of the human proteome. Science. 2015;347:1260419.

25. Tolmachev V, Grönroos TJ, Yim C-B, Garousi J, Yue Y, Grimm S, et al. Molecular design of radiocopper-labelled Affibody molecules. Sci Rep [Internet]. 2018 [cited 2019 Aug 26];8. Available from: https://www.ncbi.nlm. nih.gov/pmc/articles/PMC5916907/.

26. Rinne SS, Leitao CD, Mitran B, Bass TZ, Andersson KG, Tolmachev V, et al. Optimization of HER3 expression imaging using Affibody molecules: influence of chelator for labeling with indium-111. Sci Rep [Internet]. 2019 [cited 2019 Aug 26];9. Available from: https:/www.ncbi.nlm.nih.gov/pmc/ articles/PMC6345776/.

27. Sandberg D, Tolmachev V, Velikyan I, Olofsson H, Wennborg A, Feldwisch J, et al. Intra-image referencing for simplified assessment of HER2-expression in breast cancer metastases using the Affibody molecule ABY-025 with PET and SPECT. Eur J Nucl Med Mol Imaging. 2017:44:1337-46.

28. Johansson E, Lubberink M, Heurling K, Eriksson JW, Skrtic S, Ahlström H, et al. Whole-body imaging of tissue-specific insulin sensitivity and body composition by using an integrated PET/MR system: a feasibility study. Radiology. 2017;286:271-8.

29. Harms HJ, Knaapen P, de Haan S, Halbmeijer R, Lammertsma AA, Lubberink M. Automatic generation of absolute myocardial blood flow images using [15O]H2O and a clinical PET/CT scanner. Eur J Nucl Med Mol Imaging. 2011; 38:930-9.

\section{Publisher's Note}

Springer Nature remains neutral with regard to jurisdictional claims in published maps and institutional affiliations.

\section{Submit your manuscript to a SpringerOpen ${ }^{\circ}$ journal and benefit from:}

- Convenient online submission

- Rigorous peer review

- Open access: articles freely available online

High visibility within the field

- Retaining the copyright to your article

Submit your next manuscript at $\boldsymbol{\nabla}$ springeropen.com 